\title{
Artificial de novo biosynthesis of hydroxystyrene derivatives in a tyrosine overproducing Escherichia coli strain
}

\author{
Sun-Young Kang 1,3, Oksik Choi ${ }^{1}$, Jae Kyoung Lee ${ }^{1,3}$, Jung-Oh Ahn², Jong Seog Ahn'1, Bang Yeon Hwang ${ }^{3}$ \\ and Young-Soo Hong ${ }^{1 *}$
}

\begin{abstract}
Background: Styrene and its derivatives as monomers and petroleum-based feedstocks are valuable as raw materials in industrial processes. The chemical reaction for styrene production uses harsh reaction conditions such as high temperatures or pressures, or requires base catalysis with microwave heating. On the other hand, production of styrene and its derivatives in Escherichia coli is an environmental friendly process to produce conventional petroleum-based feedstocks.

Results: An artificial biosynthetic pathway was developed in E. coli that yields 4-hydroxystyrene, 3,4-dihydroxystyrene and 4-hydroxy-3-methoxystyrene from simple carbon sources. This artificial biosynthetic pathway has a codonoptimized phenolic acid decarboxylase (pad) gene from Bacillus and some of the phenolic acid biosynthetic genes. $E$. coli strains with the tal and pad genes, the tal, sam5, and pad genes, and the tal, sam5, com, and pad genes produced 4-hydroxystyrene, 3,4-dihydroxystyrene and 4-hydorxy-3-methoxystyrene, respectively. Furthermore, these pathways were expressed in a tyrosine overproducing E. coli. The yields for 4-hydroxystyrene, 3,4-dihydroxystyrene and 4-hydorxy-3-methoxystyrene reached 355, 63, and $64 \mathrm{mg} / \mathrm{L}$, respectively, in shaking flasks after $36 \mathrm{~h}$ of cultivation.

Conclusions: Our system is the first to use E. coli with artificial biosynthetic pathways for the de novo synthesis of 3,4-dihydroxystyrene and 4-hydroxy-3-methoxystyrene in a simple glucose medium. Similar approaches using microbial synthesis from simple sugar could be useful in the synthesis of plant-based aromatic chemicals.
\end{abstract}

Keywords: 4-Hydroxystyrene, 3,4-Dihydroxystyrene, 4-Hydroxy-3-methoxystyrene, de novo Biosynthesis

\section{Background}

Styrene is one of the most important aromatic chemicals produced industrially. It has many uses including in the manufacture of polystyrenes, plastics, and styrenebutadiene rubbers. Hydroxystyrene is also a monomer used in the production of numerous polymers and in petroleum-based feedstocks for resins, elastomers, and adhesives. Poly-hydroxystyrene, also called polyvinylphenol (PVP), is a plastic structurally similar to polystyrene. $\mathrm{PVP}$ is used in electronics as a dielectric layer in organic

\footnotetext{
*Correspondence: hongsoo@kribb.re.kr

${ }^{1}$ Chemical Biology Research Center, Korea Research Institute of Bioscience and Biotechnology, 30 Yeongudanji-ro, Ochang-eup, Chungbuk 363-883, Republic of Korea

Full list of author information is available at the end of the article
}

transistors of organic thin-film-transistor liquid-crystal display. Its ability to form linear polymers and its excellent solubility in organic solvents make hydroxystyrene a good reagent in the chemical synthesis of various coatings for electronic devices, such as a photoresist [1]. Currently, styrene production predominantly comes from the energy-intensive chemocatalytic dehydrogenation of petroleum-derived ethylbenzene $[2,3]$. Because of concerns over depleting feedstock availability and deleterious environmental impacts, a bio-based method could be a low energy, renewable alternative to petroleum-derived styrene [4]. Thus, an artificial pathway for styrene and hydroxystyrene biosynthesis from glucose in Escherichia coli was previously engineered [5-7]. 4-Hydroxystyrene is also produced in the solvent-tolerant Pseudomonas 
putida strains, originally designed for phenol and 4-coumarate production [8]. In addition, styrene production in Saccharomyces cerevisiae was recently reported combining metabolic evolution with systematic strain and pathway engineering $[9,10]$.

In recent years, several of artificial biosynthetic pathways have been engineered in microorganisms to produce useful, functionalized phenolic compounds from glucose $[4,6,11-15]$. In our laboratory, we investigate artificial biosynthetic pathways in microorganisms to produce a number of useful phenylpropanoids from plants [16-18]. Many of these substances in the phenylpropanoid pathway is phenolic acids, e.g., cinnamic, 4-coumaric, caffeic, ferulic, and sinapic acids. Their abundance has garnered much interest in their use to produced novel flavors, fragrances, pharmaceuticals and other chemicals [19].

The proposed hydroxystyrene biosynthesis pathway uses endogenously synthesized L-tyrosine as a precursor which is converted to hydroxystyrenes through a series of enzymatic steps shown in Figure 1. Phenolic acids, as key intermediates, have been previously reported for a series of phenolic acid biosynthetic genes characterized in $E$. coli [20]. The next step in the proposed hydroxystyrene biosynthesis pathway involves the subsequent decarboxylation of phenolic acids by a phenolic acid decarboxylase, which converts these acids to their styrene derivatives [21-23].
This study constructed an artificial biosynthesis pathway to produce hydroxystyrene with the tyrosine ammonia lyase gene (tal) from Saccharothrix espanaensis and phenolic acid decarboxylase gene (pad) from Bacillus amyloliquefaciens. Additionally, serial artificial biosynthetic gene expression sets were developed and used to produce 3,4-dihydroxystyrene and 4-hydorxy-3-methoxystyrene by adding 4-coumarate 3-hydroxylase gene (sam5) and caffeic acid methyltransferase gene (com), respectively. Then, an E. coli strain capable of high-level tyrosine production was constructed with the feedbackinhibition 3-deoxy-D-arabinoheptulosonate-7-phosphate (DAHP) synthase gene (aroG) and the chorismate mutase/prephenate dehydrogenase gene $(\operatorname{tyr} A)$ by modifying a previously reported expression system. This strain was highly optimized to produce phenolic acids with a heavily increased metabolic flux toward L-tyrosine. Production of both 4-hydroxystyrene and 3,4-dihydroxystyrene from glucose was about 20-fold higher in the engineered tyrosine overproducing $E$. coli strain compared to that of the wild type E. coli. Finally, the yields for 4-hydroxystyrene, 3,4-dihydroxystyrene and 4-hydorxy-3-methoxystyrene were 355,63 , and $64 \mathrm{mg} / \mathrm{L}$, respectively, in shaking flasks after $36 \mathrm{~h}$ of cultivation. This is the first report of a de novo biosynthesis yielding 3,4-dihydroxystyrene and 4-hydroxy-3-methoxystyrene using a single vector system combining phenolic acid biosynthetic genes and phenolic acid decarboxylase gene in<smiles>NC(Cc1ccc(O)cc1)C(=O)O</smiles>

TAL

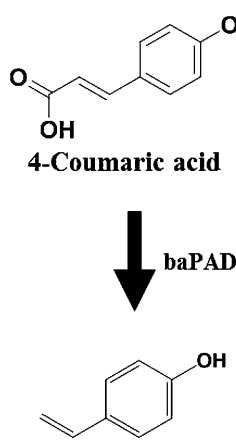

4-Hydroxystyrene

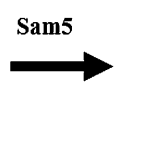<smiles>CC(C)=CC=CC(=O)O</smiles>

Caffeic acid

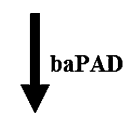<smiles>C=Cc1ccc(O)c(O)c1</smiles>

3,4-Dihydroxy styrene
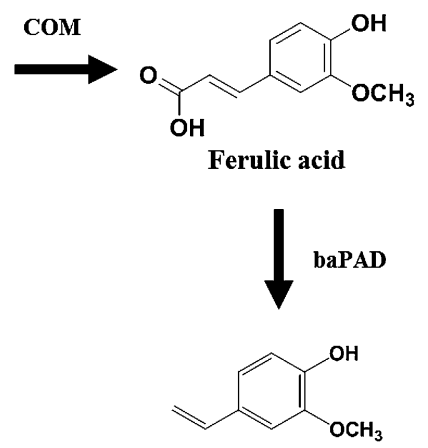

4-Hydroxy-3-methoxystyrene

Figure 1 Engineered biosynthetic pathways for hydroxystyrene derivatives starting from tyrosine in E. coli. 
E. coli. In this study, the production of 4-hydroxystyrene and its derivatives is accomplished with a carbohydrate feedstock through serial artificial biosynthetic pathways in E. coli strains.

\section{Results and discussion}

Bioconversion of phenolic acids to hydroxystyrenes through phenolic acid decarboxylase

Previous studies reported that phenolic acid decarboxylase from $B$. amyloliquefaciens produces 4-hydroxystyrene, 3,4-dihydroxystyrene and 4-hydroxy3-methoxystyrene using 4-coumaric acid, caffeic acid and ferulic acid as substrates, respectively [21]. This study investigated the functions of the codon-optimized pad gene in $E$. coli in a bioconversion experiments using cinnamic acid, 4-coumaric acid, caffeic acid, ferulic acid, and sinapic acid as substrates. The five phenolic acids were added to cultures of E. coli C41(DE3) harboring the phenolic acid decarboxylase expression vector (pET22-baPAD) to investigate whether phenolic acid decarboxylase in vivo could produce styrene derivatives (Figure 2). The culture broths and bacterial cells were collected after $36 \mathrm{~h}$ of culturing, HPLC and GC/MS analyses were done using the samples. In the bioconversion conditions, almost of the 4-coumaric acid, caffeic acid and ferulic acid was consumed in the culture media, and each hydroxystyrene was detected as a main peak on the HPLC profiles (Figure 3). The detailed results of the GC/MS analyses are presented in the additional supporting section (Additional file 1: Figure S1). When the bioconversion rate was calculated through a quantitative comparison of the feeding substrates $(2 \mathrm{mM})$ and the conversion ratios for 4-coumaric acid, caffeic acid, ferulic acid, and sinapic acid in E. coli with the pad gene were roughly $41,27,28$, and $3 \%$, respectively. In addition, the pad gene did not show any activity for cinnamic acid. These bioconversion ratios are consistent with the results of Jung et al. [21]. Interestingly, Jung et al. reported that the pad gene did not show any activity for sinapic acid. This study detected a 4-hydroxy-3,5-dimethoxystyrene peak on the HPLC profile (Figure 3D) and also confirmed its molecular weight with GC/MS analysis (Additional file 1: Figure S1). Our in vivo result for sinapic acid do not agree with the previously reported in vitro results. We do not know the reason for this, but it could be possible that the expressed PAD protein in E. coli affected the activity in vivo system.

\section{Construction of artificial biosynthetic pathways in E. coli to produce hydroxystyrene derivatives}

To produce hydroxystyrene derivatives in E. coli with a simple sugar as a substrate, a series of plasmids were

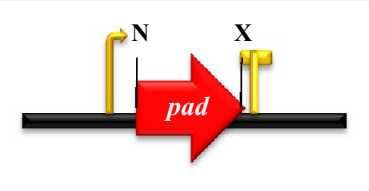

pET22-baPAD

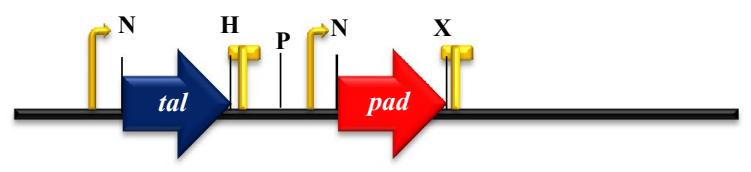

pET-opTD

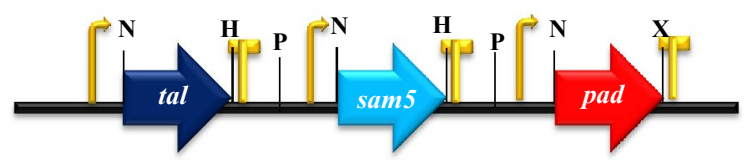

pET-opT5D

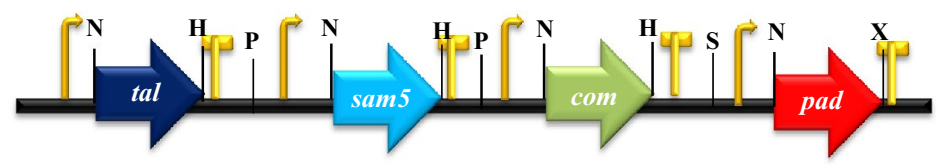

pET-opT5MD

Figure 2 Expression vectors for the phenolic acid decarboxylase pad gene (pET22-baPAD) and organization of the artificial gene clusters used to produce each hydroxystyrene derivative in E. coli. All constructs contained the T7 promoter, RBS in front of each gene, and T7 terminator at the rear of each gene. P Pacl, S Spel, N Ndel, H Hindlll, XXhol. 


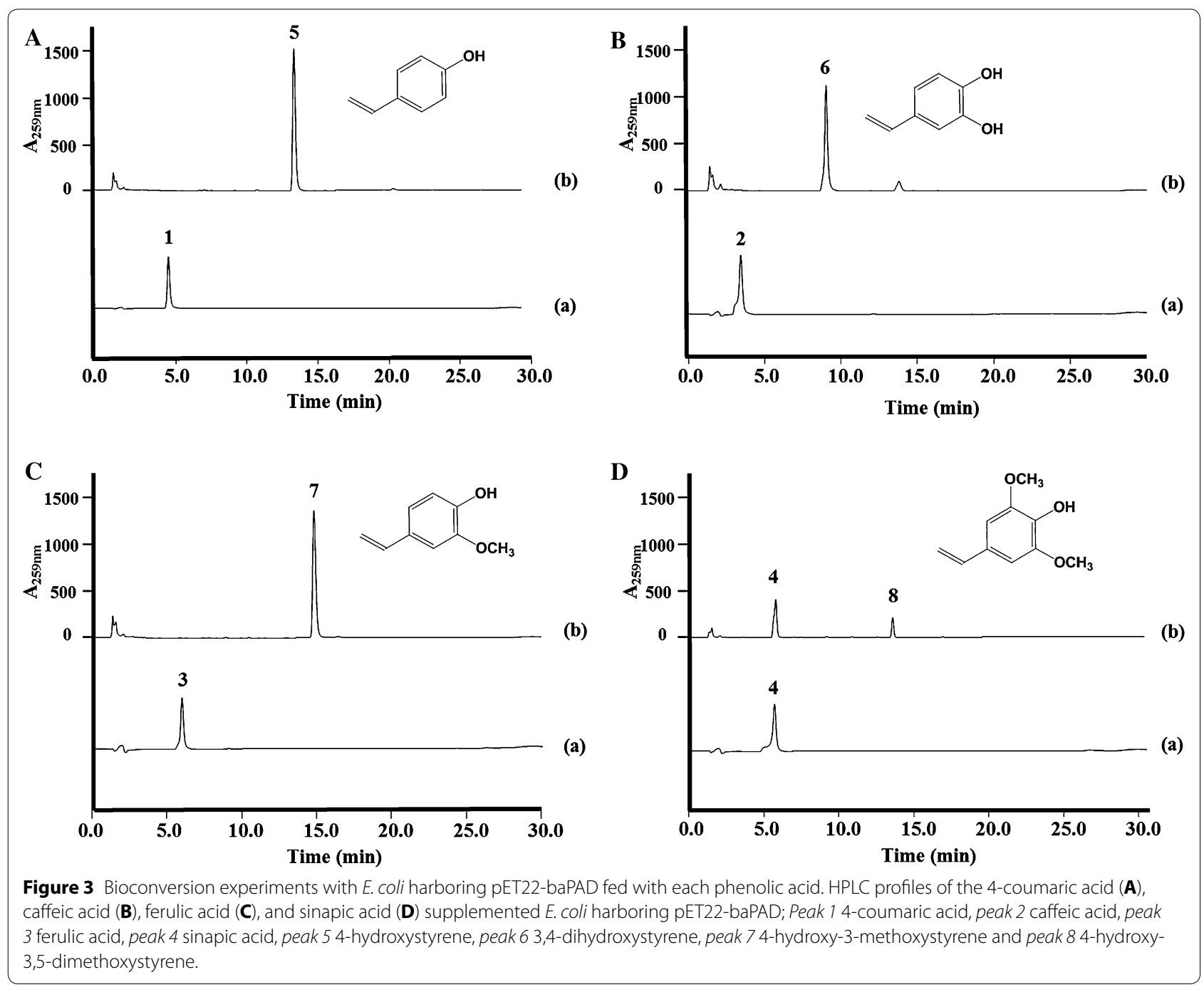

constructed containing artificial biosynthetic pathways that included the pad gene (Figure 2). We previously reported the production of 4-coumaric acid, caffeic acid, and ferulic acid in E. coli harboring artificial biosynthetic gene clusters in which the tyrosine ammonia lyase $(\mathrm{tal})$ and 4-coumarate 3-hydroxylase (sam5) from S. espanaensis and caffeic acid methyltransferase (com) from Arabidopsis thaliana [20]. The artificial biosynthetic plasmids containing the additional pad gene for the hydroxystyrene derivatives were constructed as previously described methods [17]. In this study, the pad gene was under the control of the T7 promoter. A DNA fragment containing the promoter, the pad coding region, and the terminator was amplified using the pET22-baPAD plasmid as a template. The amplified pad fragment was ligated into the pET-opTAL, -opT5 and -opT5M plasmids containing the tal gene, the tal and sam5 genes, and the tal, sam5, and com genes and designated as pET-opTD, -opT5D and -opT5MD plasmids, respectively (Figure 2). The genes each have their own T7 promoter, ribosome-binding site (RBS), and terminator sequence same as the parental vector.

E. coli cells with the artificial biosynthetic pathways were cultured in a modified synthetic medium [24] to produce hydroxystyrene derivatives. The fermentation products of the E. coli strains with the tal and pad genes, the tal, sam5 and pad genes and tal, sam5, com, and pad genes had new peaks on the HPLC profiles. The new peak at $15.0 \mathrm{~min}$ from the E. coli C41(DE3) strain with the tal and pad genes was identical to the standard for 4-hydroxystyrene, and the new peak at 9.5 min from the E. coli strain with the tal, sam5, and pad genes was identical to the standard for 3,4-dihydroxystyrene (Additional file 1: Figure S2). Contrary 
to our expectations, a peak for 4-hydroxy-3-methoxystyrene was not detected from the E. coli C41(DE3) strain with the tal, sam5, com, and pad genes after $36 \mathrm{~h}$ of culturing. Instead, an unknown peak appeared at $14 \mathrm{~min}$ on the HPLC profile, and the area of peak increased over time (Additional file 1: Figure S3). The UV spectrum of the unknown peak did not match any of the styrene derivatives including 4-hydroxystyrene and 4-hydroxy-3-methoxystyrene. However, a peak at $15.2 \mathrm{~min}$ was detected for 4-hydroxy-3-methoxystyrene after $2 \mathrm{~h}$ of culturing but disappeared after $12 \mathrm{~h}$ of culturing (Additional file 1: Figure S3). The E. coli C41(DE3) strain with the tal and pad genes (pETopTD) yielded $17.6 \pm 0.8 \mathrm{mg} / \mathrm{L}$ of 4-hydroxystyrene. The E. coli C41(DE3) strain with the tal, sam5, and pad genes (pET-opT5D) yielded $3.3 \pm 0.3 \mathrm{mg} / \mathrm{L}$ of 3,4-dihydroxystyrene (Figure 4). However, the wild type E. coli strain with the tal, sam5, com, and pad genes (pET-opT5MD) produced only a trace amount of 4-hydroxy-3-methoxystyrene after $2 \mathrm{~h}$ of culturing, and the product was not detected after $36 \mathrm{~h}$ of culturing (Figure 4).

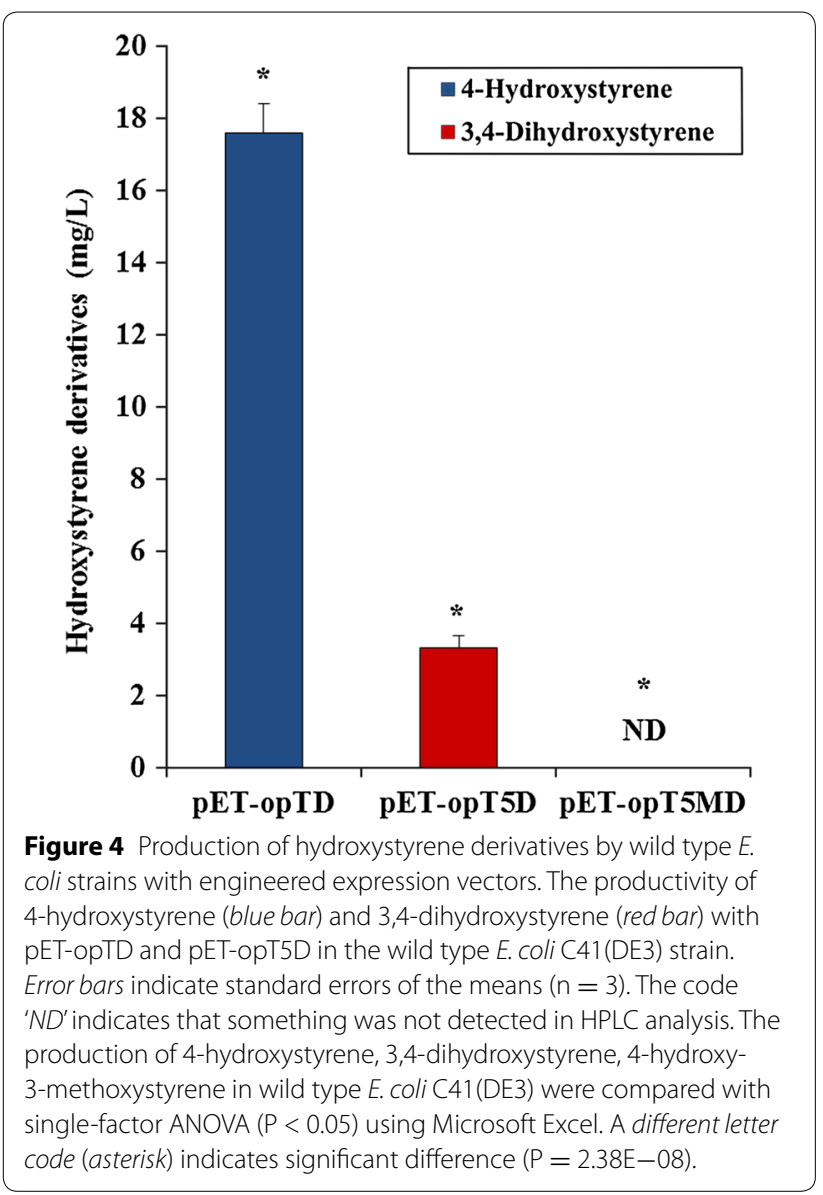

Improved production of L-tyrosine-derived phenolic acids in a tyrosine overproducing $E$. coli strain

Because tyrosine serves as an immediate endogenous precursor to the hydroxystyrene pathway, its over production in $E$. coli is essential for hydroxystyrene biosynthesis. To develop a tyrosine overproducing E. coli strain, a classical metabolic engineering strategy was used. In E. coli, the aromatic amino acid biosynthesis pathway starts with the condensation of phosphoenolpyruvate and erythrose-4-phosphate, catalyzed by 3-deoxy-D-arabinoheptulosonate-7-phosphate (DAHP) synthase present in three isoforms with each feedback-regulated by aromatic amino acids. Subsequently, L-tyrosine biosynthesis from chorismate is catalyzed by the bifunctional enzyme chorismate mutase/prephenate dehydrogenase and the aromatic amino acid transaminase. Recently, L-tyrosine excreting $E$. coli strains were produced by deregulating the aromatic amino acid biosynthesis pathway [20]. A high-copy number vector with feedbackinhibition resistant (fbr) derivatives of DAHP synthase $\left(\right.$ aro $\left.G^{f b r}\right)$ and chorismate mutase/prephenate dehydrogenase $\left(t y r A^{f b r}\right)$ genes [25] was overexpressed in an $E$. coli $\Delta$ tyrR strain [20]. In this study, the L-tyrosine producer, $E$. coli $\triangle \mathrm{COS1}$, was engineered on the genome to also overexpress the aro $G^{f b r}$ and $t y r A^{f b r}$ genes in a $\Delta$ tyrR strain background. The aro $G^{f b r}$ and $t y r A^{f b r}$ gene cassette with a strong inducible T7 promotor was inserted into the $\operatorname{tyr} R$ gene region to make a stable strain for fermentation (Additional file 1: Figures S4, S5). Overall, greater improvements in L-tyrosine production were achieved initially in wild type E. coli (Additional file 1: Figure S6). A maximum yield of $\sim 450 \mathrm{mg} / \mathrm{L}$ of L-tyrosine was produced by the E. coli $\triangle$ COS1 strain in shaken flask experiments. The tyrosine yield was comparable to the tyrosine overproducing $E$. coli strain with the $a r o G^{f b r}$ and $t y r A^{f b r}$ gene cassette vector $(400 \mathrm{mg} / \mathrm{L})$ as previously reported [20]. This $\triangle$ COS1 strain was optimized for the production of aromatic compounds resulting in a heavily increased metabolic flux towards L-tyrosine. Therefore, it is suitable platform strain for the production of other L-tyrosine-derived phenolic acids including 4-coumaric acid, caffeic acid and ferulic acid, and hydroxystyrene derivatives using the phenolic acids as precursors.

Using the same experimental conditions as above, the tyrosine-overproducing strains acquired a substantial capacity for 4-coumaric acid, caffeic acid and ferulic acid biosynthesis. As seen in Figure 4, the tyrosine overproducing E. coli $\triangle$ COS1 strain expressing the tal gene (pET-opTAL) produced more than $662 \pm 29 \mathrm{mg} / \mathrm{L}$ of 4-coumaric acid with an increase of $460 \%$ over the parental strain. The titers for caffeic acid did not improve significantly $(67 \pm 9 \mathrm{mg} / \mathrm{L})$ compared to the titers for 4-coumaric acid, despite the $478 \%$ improvement over the 
parental strain [20]. On the other hand, ferulic acid synthesis in the $\Delta$ COS1 strain expressing the tal, sam5, and com genes (pET-opT5 M) had a yield of $120 \pm 3 \mathrm{mg} / \mathrm{L}$. At the same time, an expected amount of accumulated 4-coumaric acid and caffeic acid was also identified. In agreement with previous results [20], the titers for ferulic acid were also increased over caffeic acid in the tyrosine-overproducing strain. It is possible that the metabolic pathway for ferulic acid could be alleviating any restrictions for the accumulation of caffeic acid in the cells. Finally, almost $700 \mathrm{mg} / \mathrm{L}$ of 4 -coumaric acid was produced. Maximum titers of 76 and $123 \mathrm{mg} / \mathrm{L}$ for caffeic acid and ferulic acid were achieved, respectively (Figure 5). Thus, the results of this study suggest that in a tyrosine-overproducing strain system, higher titers of artificial phenolic acids from the biosynthetic pathways mentioned above guarantee higher concentrations of hydroxystyrene derivatives.

\section{Improved production of hydroxystyrene derivatives in tyrosine overproducing strains}

Next, the fermentation products of the E. coli C41(DE3) strain and the tyrosine-overproducing $\triangle \mathrm{COS} 1$ strain were compared each with the tal and pad (pET-opTD), tal, sam5 and pad (pET-opT5D), and tal, sam5, com, and pad (pET-opT5MD) genes, respectively. With the same experimental conditions, the overproducingtyrosine strains had a substantial synthesis capacity for 4-hydroxystyrene, 3,4-dihydroxystyrene and 4-hydroxy3-methoxystyrene. As seen in Figure 6, the $\triangle \mathrm{COS} 1$ strain expressing the tal and pad genes (pET-opTD) produced $355 \mathrm{mg} / \mathrm{L}$ of 4-hydroxystyrene compared to the $17.6 \mathrm{mg} / \mathrm{L}$ produced in the C41(DE3) strain with the same genes. The $\triangle \mathrm{COS} 1$ strain expressing the tal, sam5 and pad genes (pET-opT5D) produced $63 \mathrm{mg} / \mathrm{L}$ of 3,4-dihydroxystyrene compared to the $3.3 \mathrm{mg} / \mathrm{L}$ produced in the C41(DE3) strain with the same genes. Interestingly, $64 \mathrm{mg} / \mathrm{L}$ of 4-hydroxy-3-methoxystyrene was produced in only the $\triangle \mathrm{COS} 1$ strain expressing the tal, sam5, com, and pad genes (pET-opT5MD) with no yields in the C41(DE3) strain expressing the same genes after $36 \mathrm{~h}$ of culturing (Figure 4). An unknown peak at $14 \mathrm{~min}$ was also detected in addition to the 4-hydroxy-3-methoxystyrene peak from the $\triangle$ COS1 strain. At the same time, a small amount (12 and $4 \mathrm{mg} / \mathrm{L}$ ) of accumulated 4-hydroxystyrene and 3,4-dihydroxystyrene, respectively,
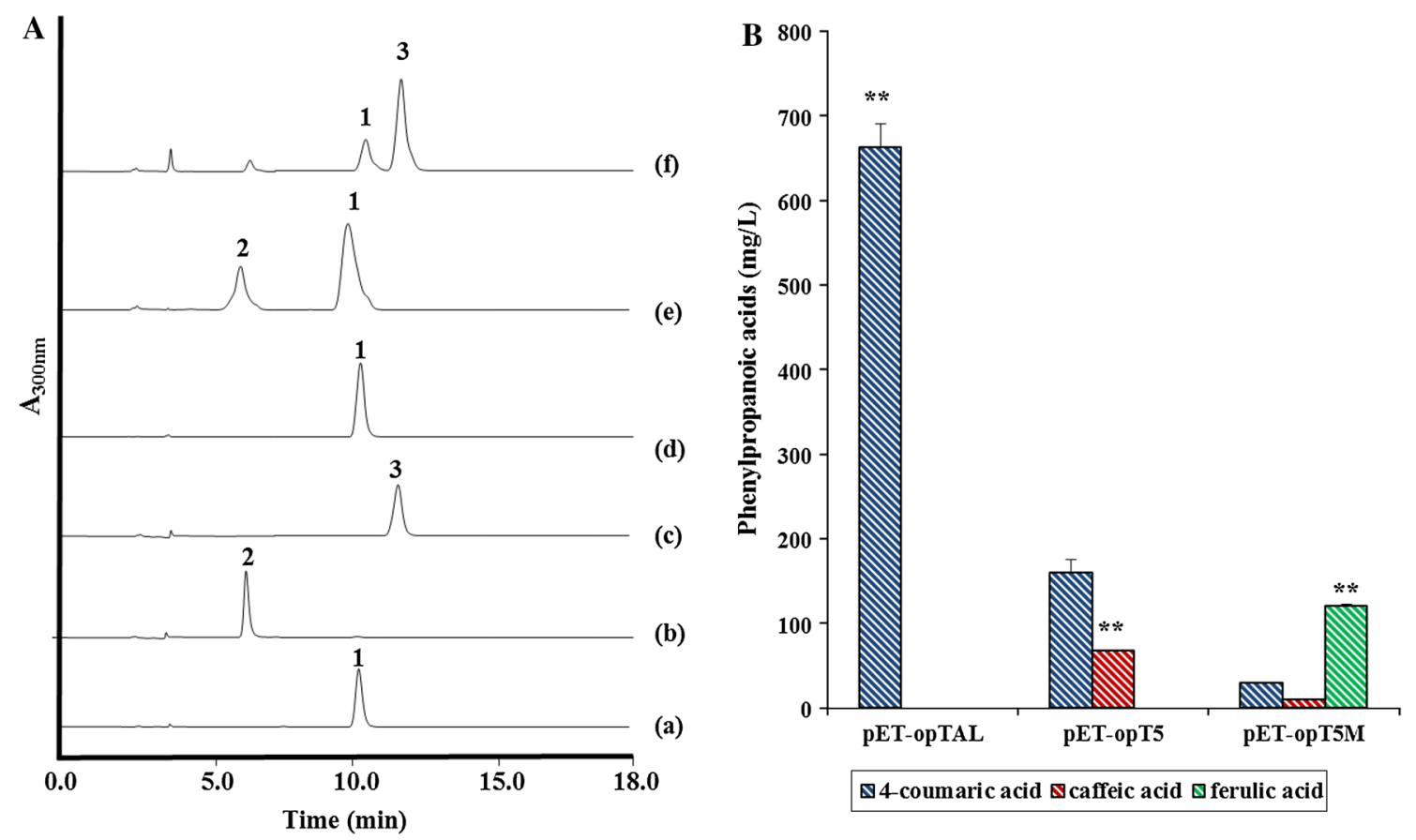

Figure 5 Production of phenolic acids by engineered tyrosine overproducing $E$. coli $\triangle$ COS1 with engineered expression vectors. A HPLC profile of the standard 4-coumaric acid (a), caffeic acid (b), and ferulic acid (c); the culture broth of tyrosine overproducing E. coli $\triangle$ COS1 harboring pET-opTAL (d); pET-opT5 (e); pET-opT5 M (f). The absorbance was monitored at $300 \mathrm{~nm}$. Peak 14 -coumaric acid, peak 2 caffeic acid, peak 3 ferulic acid. B The production of 4-coumaric acid (diagonal blue), caffeic acid (diagonal red), ferulic acid (diagonal green) with pET-opTAL, pET-opT5 and pET-opT5 M in mutant $\Delta \operatorname{COS} 1$ strain. Error bars indicate standard errors of the means $(n=3)$. The production of 4-coumaric acid, caffeic acid and ferulic acid in $\triangle$ COS1 were compared with single-factor ANOVA $(P<0.05)$ using Microsoft Excel. A different letter code (double asterisk) indicates significant difference $(P=2.25 E-08)$. 

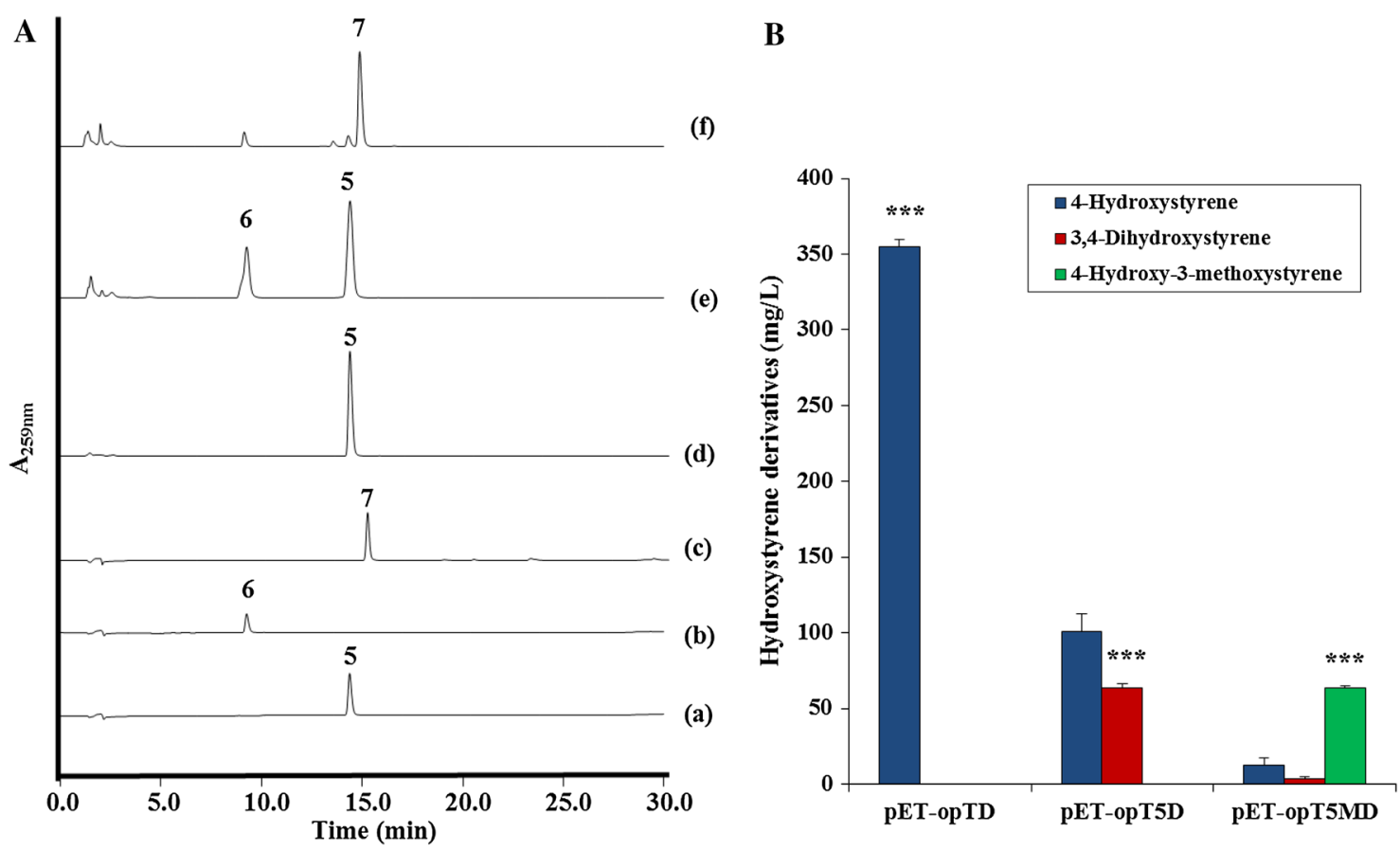

Figure 6 Production of hydroxystyrene derivatives by engineered tyrosine overproducing E. coli $\Delta$ COS1 with engineered expression vectors. A HPLC profiles of standard 4-hydroxystyrene (a); 3,4-dihydroxystyrene (b); 4-hydroxy-3-methoxystyrene (c); the culture broth of tyrosine overproducing E. coli harboring pET-opTD (d); pET-opT5D (e); pET-opT5MD (f). The absorbance was monitored at $259 \mathrm{~nm}$. Peak 5 4-hydroxystyrene, peak 63,4-dihydroxystyrene, peak 74-hydroxy-3-methoxystyrene. B The productivity of 4-hydroxystyrene (blue bar), 3,4-dihydroxystyrene (red bar), and 4-hydroxy-3-methoxystyrene (green bar) with pET-opTD, pET-opT5D, and pET-opT5MD in $\Delta$ COS1 strain. Error bars indicate standard errors of the means $(n=3)$. The production of 4-hydroxystyrene, 3,4-dihydroxystyrene, 4-hydroxy-3-methoxystyrene in $\Delta$ COS1 were compared with singlefactor ANOVA ( $P$ < 0.05) using Microsoft Excel. A different letter code (triple asterisk) indicates significant difference $(P=5.66 \mathrm{E}-11)$.

was also identified in the $\triangle$ COS1 strain expressing the tal, sam5, com, and pad genes (pET-opT5MD). To achieve higher yields of the hydroxystyrene derivatives, metabolite pattern analyses were performed for the tyrosine-overproducing $\triangle \mathrm{COS} 1$ strains expressing the tal and pad (pET-opTD), tal, sam5 and pad (pET-opT5D), and tal, sam5, com, and pad genes (pET-opT5MD) according to culture times at 120,40 and $50 \mathrm{~h}$, respectively, until the production of each hydroxystyrene derivative was saturated. The amount of 4-hydroxystyrene was about $572 \mathrm{mg} / \mathrm{L}$ at $120 \mathrm{~h}$ while that for 3,4-dihydroxystyrene and 4-hydroxy-3-methoxystyrene were about $65 \mathrm{mg} / \mathrm{L}$ at $40 \mathrm{~h}$ and $64 \mathrm{mg} / \mathrm{L}$ at $36 \mathrm{~h}$, respectively (Additional file 1: Figure S7). The production of 4-hydroxystyrene and 3,4-dihydroxystyrene increased by 20 -fold and 21-fold compared to the parental strains, respectively. Furthermore, only the $\triangle \mathrm{COS} 1$ strain expressing the tal, sam5, com, and pad genes (pET-opT5MD) produced $64 \mathrm{mg} / \mathrm{L}$ of 4-hydroxy-3-methoxystyrene with no yields in the C41(DE3) strain expressing the same genes. When comparing the established baseline production of 4-hydroxystyrene from a previously reported $E$. coli platform that used corn cobs as a renewable substrate [26,27], the titers and yields achieved in this study were about 2-fold less. However, the titers of 4-hydroxystyrene in this study were achieved with a simple glucose medium as a substrate in culture conditions using shake flasks. The results suggest that system used in this study as well as the achieved titers forms the basis for further improvement in the production of hydroxystyrene derivatives, and opens the possibility for a jar fermentation and extraction process. Furthermore, the previous report showed that the maximum concentration of 4-hydroxystyrene was limited to $3.3 \mathrm{mM}$ because of the cytotoxicity of the product to the $E$. coli host $[5,28]$. To alleviate the cytotoxicity of intermediates (phenolic acids) or products (hydroxystyrenes), a new host, such as solvent tolerant microbes, could be the solution for higher titers. For example, the solventtolerant P. putida S12 through a two-phase fermentation had higher than $10 \mathrm{~g} / \mathrm{L}$ yields of 4-hydroxystyrene [8]. Further, if our approach described in this manuscript applies to the engineering of solvent tolerant microbes, such as Pseudomonas and yeast platforms, higher titer could be achieved [8, 9]. Furthermore, a fermentation platform using low-priced precursor sources, such agroindustrial wastes and lignocellulose materials [5, 27, 29], 
could be what is needed for the substantial and economical production of hydroxystyrene monomers, which are presently produced from non-renewable petroleum.

\section{Conclusions}

The system in this study converts 4-coumaric acid to 4-hydroxystyrene through pad (phenolic acid decarboxylase), which is eventually converted caffeic acid and ferulic acid and then to 3,4-dihydroxystyrene and 4-hydorxy3-methoxystyrene, respectively. E. coli strains expressing tal (tyrosine ammonia lyase) and pad, and tal, sam5 (4-coumarate 3-hydroxylase), and pad, and tal, sam5, com (caffeic acid methyltransferase), and pad produced 4-hydroxystyrene, 3, 4-dihydroxystyrene and 4-hydroxy3-methoxystyrene, respectively, using a simple sugar medium without precursor feeding. Furthermore, these pathways were extended in an E. coli strain with the biosynthesis machinery for overproducing tyrosine. Finally, the titers for 4-hydroxystyrene, 3,4-dihydroxystyrene and 4-hydroxy-3-methoxystyrene in the tyrosine-overproducing $E$. coli strains reached 355,63 , and $64 \mathrm{mg} / \mathrm{L}$, respectively, in shaking flasks after $36 \mathrm{~h}$ of cultivation.

\section{Methods}

Bacterial strains, plasmids, and chemicals

The strains and plasmids used in this study are listed in Table 1. Antibiotics were added to the medium as required at the following concentrations: ampicillin, $100 \mathrm{mg} / \mathrm{L}$; kanamycin, $50 \mathrm{mg} / \mathrm{L}$; and tetracycline, $10 \mathrm{mg} / \mathrm{L}$. Cinnamic acid, 4-coumaric acid, caffeic acid, ferulic acid, sinapic acid, 4-hydroxystyrene (4-vinylphenol), and 4-hydroxy-3-methoxystyrene (2-methoxy-4-vinylphenol) were purchased from Sigma-Aldrich (USA), and 3,4-dihydroxystyrene was purchased from Toronto Research Chemicals Inc. (Canada) as a standard for compound identification by HPLC.

\section{DNA manipulation}

The restriction enzymes (NEB; Takara), Ex Taq polymerase (Takara), pfu Taq polymerase (Enzynomics, Korea), an AccuPower Ligation kit (Bioneer, Korea), and a Quick \& Easy E. coli gene deletion kit (Gene Bridges, German) were used according to the manufacturers' instructions. The optimized tyrosine ammonia lyase gene $(t a l)$ and 4-coumarate 3-hydroxylase gene (sam5) from S. espanaensis and the caffeic acid methyltransferase gene (com) from $A$. thaliana were synthesized previously by DNA 2.0 [20]. Codon optimization and synthesis of the phenolic acid decarboxylase gene ( $p a d)$ from B. amyloliquefaciens DSM7 (GenBank FN597644) were performed with the GeneGPS ${ }^{\mathrm{mm}}$ program (DNA2.0).

\section{Construction of phenolic acid decarboxylase expression vectors and assembly of the artificial biosynthetic pathways}

In order to construct an expression vector containing the phenolic acid decarboxylase gene ( $p a d)$ that was under

\section{Table 1 Plasmids and strains used in this study}

\begin{tabular}{|c|c|c|}
\hline Plasmid or strain & Relevant characteristics & Source \\
\hline \multicolumn{3}{|l|}{ Plasmid } \\
\hline pET-22b(+) & f1 ori, T7 promoter, Amp ${ }^{R}$ & Novagen \\
\hline pET-28a $(+)$ & f1 ori, T7 promoter, Kan ${ }^{R}$ & Novagen \\
\hline pET-opTAL & pET-28a $(+)$ carrying codon-optimized tyrosine ammonia lyase gene (tal) & Kang et al. [20] \\
\hline pET-Sam5 & pET-28a(+) carrying 4-coumarate 3-hydroxylase gene (sam5) & Choi et al. [17] \\
\hline pET-COM & pET-28a $(+)$ carrying caffeic acid methyltransferase gene $(c o m)$ & Choi et al. [17] \\
\hline pET-opT5 & pET-28a $(+)$ carrying tal and sam5 gene & Kang et al. [20] \\
\hline pET-opT5M & pET-28a(+) carrying tal, sam5, and com gene & Kang et al. [20] \\
\hline pET28-tyrA* & $\begin{array}{l}\text { pET-28a(+) carrying feedback-inhibition resistant chorismate mutase/prephenate dehydrogenase gene } \\
\left(\mathrm{tyrA}^{\mathrm{fbr}}\right)\end{array}$ & Kang et al. [20] \\
\hline pET22-aroG ${ }^{*}$ & pET-22b(+) carrying feedback-inhibition resistant DAHP synthase gene ( rroG $^{\mathrm{fbr}}$ ) & Kang et al. [20] \\
\hline pET-AG & pET-28a $(+)$ carrying tyrA ${ }^{\mathrm{fbr}}$ and aroG $^{\mathrm{fbr}}$ gene & This study \\
\hline pET22-baPAD & pET-22b(+) carrying codon-optimized phenolic acid decarboxylase gene (pad) & This study \\
\hline pET-opTD & pET-28a $(+)$ carrying tal and pad gene & This study \\
\hline pET-opT5D & pET-28a $(+)$ carrying tal, sam5 and pad gene & This study \\
\hline pET-opT5MD & pET-28a $(+)$ carrying tal, sam5, com and pad gene & This study \\
\hline \multicolumn{3}{|l|}{ Strain } \\
\hline E. coli DH5 $\alpha$ & Cloning host & Invitrogen \\
\hline E. coli C41(DE3) & Derivative strain of E. coli BL21(DE3) & Miroux and Walker [31] \\
\hline$\Delta$ tyrR & tryR gene in-frame deletion mutant of E. coli C41(DE3) & Kang et al. [20] \\
\hline$\Delta \cos 1$ & E. coli C41(DE3); $\Delta$ tyrR::tyrA ${ }^{\mathrm{fbr}}$, aro $\mathrm{G}^{\mathrm{fbr}}$; tyrosine overproducing strain & This study \\
\hline
\end{tabular}


the control of independent T7 promoter, a 0.5-kb DNA fragment, which contained the synthetic pad coding region, was cloned into the NdeI and XhoI sites on pET$22 \mathrm{~b}(+)$, which resulted in pET22-baPAD. The three genes (tal, sam5, and com) were independently cloned into $\mathrm{pET}-22 \mathrm{~b}(+)$ or $\mathrm{pET}-28 \mathrm{a}(+)$ vectors $[17,20]$. Using the tal, sam5, com, and pad genes as templates, four DNA fragments were amplified by PCR with the appropriate pairs of primers. In order to assemble the pET-opTD vector, the tal coding region was amplified using pETopTAL as a template with the primer opTAL-F (5'-CAT ATGACCCAGGTGGTTGAACGCC-3') and Cpac (the sequence is located downstream of the T7 terminator region of the $\mathrm{PET}$ vector and contains the designed $\mathrm{PacI}$ site: TTAATTAATGCGCCGCTACAGGGCGCGTCC), also the pad coding region was amplified using pET22baPAD as a template with the primer Npac (the sequence is located upstream of the T7 promoter region of the pET vector and contains the designed PacI site: TTAAT TAATCGCCGCGACAATTTGCGACGG) and baPAD-R (the sequence contains the designed XhoI site $5^{\prime}$-CTC GAGTTACTTCAGTTTACC-3'). Each of the amplified fragments were digested with corresponding sites and cloned between the NdeI and XhoI digested pET-28a $(+)$ via ligation, which resulted in pET-opTD. A 2.5-kb PacI fragment containing the sam5 gene was PCR-amplified with the NPac and CPac primers using pET22-Sam5 as a template. The amplified fragment was digested with $P a c \mathrm{I}$ and cloned between the PacI digested pET-opTD, which resulted in pET-opT5D. Finally, a $2.5-\mathrm{kb}$ PacI/SpeI fragment containing the com gene was PCR-amplified with the NPac and Cspe (the sequence is located downstream of the T7 terminator region of the pET vector and contains the designed SpeI site: ACTAGTTCCTCCTTTCAGCAAAAAACCCCTC) primers using pET22$\mathrm{COM}$ as a template. A $2.5-\mathrm{kb}$ PacI fragment containing the sam5 gene was PCR-amplified with the NPac and CPac primers using pET22- Sam5 as a template. Each of the two amplified fragments were digested with corresponding sites and cloned into PacI digested pET-opTD, which resulted in pET-opT5MD (Figure 2).

\section{Construction of the L-tyrosine overproducing strain}

An L-tyrosine over-producing strain of $E$. coli $(\triangle \mathrm{COS} 1)$ was achieved by extra gene insertion of aroG and tyrA, feedback-inhibition resistance (fbr) genes on the tyrR gene locus. The genetic design of the aro $G$ and tyr $A$ feedback-inhibition resistance (fbr) genes was followed as previously described by Lütke-Eversloh and Stephanopoulos $[25,30]$ and used our previously made constructs [20]. The PCR product was generated using the tyr $A^{f b r}$-aro $G^{f b r}$-FRT-neo-FRT fragment as a template for pET-AG and FRT-neo-FRT fragment (Gene Bridges). We made a fragment containing both the $t y r A^{f b r}$ and $a r o G^{f b r}$ gene cassette, in which the RBS and T7 promoter were positioned in front of each gene, through PCR with the following primers, IF-N1(5'-CGGTACCCGGGGATCAC TAGTTGATCGGCGCGAGATTTAATCGCCGCGCAA T-3') and IF-C1(5'-GTTAATTAAACTAGTCACGCT GCGCGTAACCACCACACCCGC CGCGCT-3'), and another fragment containing the FRT-neo-FRT using the following primers, IF-FRT1(5'-ACTAGTTTAATTAACC CTCACTAAAGGGCGGCCGCGAAGTTCCTATT-3') and IF-FRT2(5'-CGACTCTAGAGGATCACTAGTAAT ACGACTCACTATAGGGCTCGAG GAAGTTCC-3'). These two fragments were connected between the SpeI site of pUC19 using the In-fusion kit (Clontech Laboratories, Inc., USA), resulting in pUC-AGFRT (Additional file 1: Figure S4). The 5.9-kb insertion PCR product was generated using pUC-AGFRT as a template and the following primers, tyrRr (5'-ATCAGGCAT ATTCGCGCTTACTCTT CGTTCTTCTTCTGACTCA GACCATATAATACG ACTCACTATAGGGCTC-3') and Inf-tryRfAG (5'-GTCATATCATCATATTAATT GTTCTTTTTTCAGGTGAAGGTTCCCATGC GTAC TAGTCGTTCTACCATCGACACC-3'), and this gene cassette was inserted between the tyrR gene for gene insertional inactivation, which was done as previously reported using RED/ET recombination with a Quick \& Easy E. coli Gene deletion kit (Gene Bridges). The clones growing on the kanamycin plate still contained the selection marker cassette, while all other clones containing insertional inactivation lost the selection marker. The kanamycin selection marker was removed from the chromosome by transforming the cells with an FLP recombinase expression plasmid, 707-FLPe (Gene Bridges). The insertional inactivation mutant $(\triangle \mathrm{COS} 1)$ was verified through PCR using the following primers: tyrA-F ( $5^{\prime}-$ CCATGGTTGCTGAATTGACCGCATTACG-3') and aroG-R (5'- AAGCTTAACCACGA CGCGCTTTCA CAGC- $3^{\prime}$ ). The PCR product was sequenced and verified (Additional file 1: Figure S5).

\section{Culture conditions for production}

Recombinant E. coli C41 (DE3) strains [31] harboring plasmids were grown at $37^{\circ} \mathrm{C}$ in a Luria-Bertani (LB) medium containing $50 \mu \mathrm{g} / \mathrm{mL}$ kanamycin. The overnight culture was inoculated (1.5\%) into fresh LB medium supplemented with the same concentration of kanamycin. The culture was grown at $37^{\circ} \mathrm{C}$ to an optical density at $600 \mathrm{~nm}\left(\mathrm{OD}_{600}\right)$ of 0.6 , and IPTG was added to the final concentration of $1 \mathrm{mM}$, and the culture was incubated for $6 \mathrm{~h}$. The cells were harvested by centrifugation, suspended, and incubated at $26^{\circ} \mathrm{C}$ for $36 \mathrm{~h}$ in a modified synthetic medium $\left(3 \mathrm{~g} / \mathrm{L} \mathrm{KH}_{2} \mathrm{PO}_{4}, 7.3 \mathrm{~g} / \mathrm{L} \mathrm{K}_{2} \mathrm{HPO}_{4}\right.$, 8.4 g/L MOPS, $2 \mathrm{~g} / \mathrm{L} \mathrm{NH}_{4} \mathrm{Cl}, 0.5 \mathrm{~g} / \mathrm{L} \mathrm{NaCl}, 0.1 \mathrm{ml} / \mathrm{L}$ Trace 
elements, $5 \mathrm{~g} / \mathrm{L}\left(\mathrm{NH}_{4}\right)_{2} \mathrm{SO}_{4}, 5 \mathrm{~g} / \mathrm{L} \mathrm{MgSO}_{4}$, and supplemented with $15 \mathrm{~g} / \mathrm{L}$ glucose, $1 \mathrm{mM}$ IPTG and appropriate antibiotics) [24]. For the feeding experiments, the cultures were supplemented with cinnamic acid, 4-coumaric acid, caffeic acid, ferulic acid, or sinapic acid (final concentration: $2 \mathrm{mM}$ ), respectively. The samples were collected after $36 \mathrm{~h}$ and analyzed by HPLC.

\section{Detection and quantification of the products}

To quantify 4-hydroxystyrene, 3,4-dihydroxystyrene and 4-hydroxy-3-methoxystyrene, $1 \mathrm{~mL}$ of cell-free culture supernatants was filtered through a $0.2 \mu \mathrm{m}$ cellulose membrane syringe filter (Sartorius) and used for HPLC analysis with a Dionex Separations module connected with a Photodiode Array detector (Dionex) set. Twenty microliters of the samples were applied to a J'sphere ODSH80 column $(4.6 \times 150 \mathrm{~mm}$ i.d., $5 \mu \mathrm{m}$; YMC, Japan $)$ in a high-performance liquid chromatography (HPLC) system $\left[\mathrm{CH}_{3} \mathrm{CN}-\mathrm{H}_{2} \mathrm{O}\right.$ (0.05\% trifluoroacetic acid), $20-60 \%$ acetonitrile $\left(\mathrm{CH}_{3} \mathrm{CN}\right)$ for $25 \mathrm{~min}$ at flow rate of $1 \mathrm{~mL} / \mathrm{min}$; Dionex, USA] equipped with a photodiode array detector. Quantification of the three above-mentioned compounds was based on the peak areas of absorbance at $259 \mathrm{~nm}$. Purchased 3,4-dihydroxystyrene and 4-hydroxy-3-methoxystyrene as a standard contains the impurity. Therefore, all the hydroxystyrene derivatives were measured as equivalent to 4-hydroxystyrene. For the quantification of 4-coumaric acid, caffeic acid, and ferulic acid, the HPLC analysis was followed as our previously described methods [20]. The data shown in this study were generated from triplicate independent experiments. The titers for each production were compared with single-factor ANOVA $(\mathrm{P}<0.05)$ using the single-factor ANOVA tool.

\section{Additional files}

Additional file 1: Further details of relevance to this study.

\begin{abstract}
Authors' contributions
SK and OC performed the experiments and wrote the manuscript. JL coperformed the experiments on the metabolite analysis. $\mathrm{BH}$, JOA and JSA contributed general advice, particularly on the metabolite analysis and resource support. YH designed all the experiments and wrote the manuscript. All authors read and approved the final manuscript.
\end{abstract}

\section{Author details \\ ${ }^{1}$ Chemical Biology Research Center, Korea Research Institute of Bioscience and Biotechnology, 30 Yeongudanji-ro, Ochang-eup, Chungbuk 363-883, Republic of Korea. ${ }^{2}$ Biotechnology Process Engineering Center, Korea Research Institute of Bioscience and Biotechnology, 30 Yeongudanji-ro, Ochang-eup, Chungbuk 363-883, Republic of Korea. ${ }^{3}$ Department of Phar- macy Graduate School, Chungbuk National University, Cheongju 361-763, Republic of Korea.}

\section{Acknowledgements}

This work was supported in part by Global R\&D Center program, NRF and by the Next-Generation BioGreen 21 Program (SSAC, PJ011084012015),
RDA Republic of Korea. We thank the Gyeonggi Bio Center for the GC/MS measurements.

\section{Compliance with ethical guidelines}

\section{Competing interests}

The authors declare that they have no competing interests.

Received: 8 April 2015 Accepted: 25 May 2015

Published online: 10 June 2015

\section{References}

1. Bernini R, Mincione E, Barontini M, Provenzanoa GLS (2007) Obtaining 4-vinylphenols by decarboxylation of natural 4-hydroxycinnamic acids under microwave irradiation. Tetrahedron 63:9663-9667

2. Diao J, Liu H, Wang J, Feng Z, Chen T, Miao C et al (2015) Porous graphene-based material as an efficient metal free catalyst for the oxidative dehydrogenation of ethylbenzene to styrene. Chem Commun 51:3423-3425

3. Keller N, Maksimova NI, Roddatis W, Schur M, Mestl G, Butenko YV et al (2002) The catalytic use of onion-like carbon materials for styrene synthesis by oxidative dehydrogenation of ethylbenzene. Angew Chem Int Ed Engl 41:1885-1888

4. Claypool JT, Raman DR, Jarboe LR, Nielsen DR (2014) Technoeconomic evaluation of bio-based styrene production by engineered Escherichia coli. J Ind Microbiol Biotechnol 41:1211-1216

5. Qi WW, Vannelli T, Breinig S, Ben-Bassat A, Gatenby AA, Haynie SL et al (2007) Functional expression of prokaryotic and eukaryotic genes in Escherichia coli for conversion of glucose to p-hydroxystyrene. Metab Eng 9:268-276

6. Sariaslani FS (2007) Development of a combined biological and chemical process for production of industrial aromatics from renewable resources. Annu Rev Microbiol 61:51-69

7. McKenna R, Nielsen DR (2011) Styrene biosynthesis from glucose by engineered E. coli. Metab Eng 13:544-554

8. Verhoef S, Wierckx N, Westerhof RG, de Winde JH, Ruijssenaars HJ (2009) Bioproduction of $p$-hydroxystyrene from glucose by the solvent-tolerant bacterium Pseudomonas putida S12 in a two-phase water-decanol fermentation. Appl Environ Microbiol 75:931-936

9. McKenna R, Thompson B, Pugh S, Nielsen DR (2014) Rational and combinatorial approaches to engineering styrene production by Saccharomyces cerevisiae. Microb Cell Fact 13:123

10. McKenna R, Moya L, McDaniel M, Nielsen DR (2015) Comparing in situ removal strategies for improving styrene bioproduction. Bioprocess Biosyst Eng 38:165-174

11. Adkins J, Pugh S, McKenna R, Nielsen DR (2012) Engineering microbial chemical factories to produce renewable "biomonomers". Front Microbiol $3: 313$

12. Woolston BM, Edgar S, Stephanopoulos G (2013) Metabolic engineering: past and future. Annu Rev Chem Biomol Eng 4:259-288

13. Jang YS, Kim B, Shin JH, Choi YJ, Choi S, Song CW et al (2012) Biobased production of C2-C6 platform chemicals. Biotechnol Bioeng 109:2437-2459

14. Lee JW, Na D, Park JM, Lee J, Choi S, Lee SY (2012) Systems metabolic engineering of microorganisms for natural and non-natural chemicals. Nat Chem Biol 8:536-546

15. Barker JL, Frost JW (2001) Microbial synthesis of $p$-hydroxybenzoic acid from glucose. Biotechnol Bioeng 76:376-390

16. Choi O, Lee JK, Kang SY, Pandey RP, Sohng JK, Ahn JS et al (2014) Construction of artificial biosynthetic pathways for resveratrol glucoside derivatives. J Microbiol Biotechnol 24:614-618

17. Choi O, Wu CZ, Kang SY, Ahn JS, Uhm TB, Hong YS (2011) Biosynthesis of plant-specific phenylpropanoids by construction of an artificial biosynthetic pathway in Escherichia coli. J Ind Microbiol Biotechnol 38:1657-1665

18. Kang SY, Lee JK, Choi O, Kim CY, Jang JH, Hwang BY et al (2014) Biosynthesis of methylated resveratrol analogs through the construction of an artificial biosynthetic pathway in E. coli. BMC Biotechnol 14:67 
19. Winkel-Shirley B (2001) Flavonoid biosynthesis. A colorful model for genetics, biochemistry, cell biology, and biotechnology. Plant Physiol 126:485-493

20. Kang SY, Choi O, Lee JK, Hwang BY, Uhm TB, Hong YS (2012) Artificial biosynthesis of phenylpropanoic acids in a tyrosine overproducing Escherichia coli strain. Microb Cell Fact 11:153

21. Jung DH, Choi W, Choi KY, Jung E, Yun H, Kazlauskas RJ et al (2013) Bioconversion of $p$-coumaric acid to $p$-hydroxystyrene using phenolic acid decarboxylase from B. amyloliquefaciens in biphasic reaction system. Appl Microbiol Biotechnol 97:1501-1511

22. Mukai N, Masaki K, Fujii T, Kawamukai M, lefuji H (2010) PAD1 and FDC1 are essential for the decarboxylation of phenylacrylic acids in Saccharomyces cerevisiae. J Biosci Bioeng 109:564-569

23. Cavin JF, Barthelmebs L, Divies C (1997) Molecular characterization of an inducible $p$-coumaric acid decarboxylase from Lactobacillus plantarum: gene cloning, transcriptional analysis, overexpression in Escherichia coli, purification, and characterization. Appl Environ Microbiol 63:1939-1944

24. Zhang H, Stephanopoulos G (2013) Engineering E. coli for caffeic acid biosynthesis from renewable sugars. Appl Microbiol Biotechnol 97:3333-3341

25. Lutke-Eversloh T, Stephanopoulos G (2005) Feedback inhibition of chorismate mutase/prephenate dehydrogenase (TyrA) of Escherichia coli: generation and characterization of tyrosine-insensitive mutants. Appl Environ Microbiol 71:7224-7228
26. Salgado JM, Rodriguez-Solana R, Curiel JA, de Las Rivas B, Munoz R, Dominguez JM (2014) Bioproduction of 4-vinylphenol from corn cob alkaline hydrolyzate in two-phase extractive fermentation using free or immobilized recombinant E. coli expressing pad gene. Enzyme Microb Technol 58-59:22-28

27. Salgado JM, Rodriguez-Solana R, Curiel JA, de Las Rivas B, Munoz R, Dominguez JM (2012) Production of vinyl derivatives from alkaline hydrolysates of corn cobs by recombinant Escherichia coli containing the phenolic acid decarboxylase from Lactobacillus plantarum CECT 748T. Bioresour Technol 117:274-285

28. Ramos-Gonzalez MI, Ben-Bassat A, Campos MJ, Ramos JL (2003) Genetic engineering of a highly solvent-tolerant Pseudomonas putida strain for biotransformation of toluene to $p$-hydroxybenzoate. Appl Environ Microbiol 69:5120-5127

29. Coghe S, Benoot K, Delvaux F, Vanderhaegen B, Delvaux FR (2004) Ferulic acid release and 4-vinylguaiacol formation during brewing and fermentation: indications for feruloyl esterase activity in Saccharomyces cerevisiae. J Agric Food Chem 52:602-608

30. Lutke-Eversloh T, Stephanopoulos G (2007) L-tyrosine production by deregulated strains of Escherichia coli. Appl Microbiol Biotechnol 75:103-110

31. Miroux B, Walker JE (1996) Over-production of proteins in Escherichia coli: mutant hosts that allow synthesis of some membrane proteins and globular proteins at high levels. J Mol Biol 260:289-298

\section{Submit your next manuscript to BioMed Central and take full advantage of:}

- Convenient online submission

- Thorough peer review

- No space constraints or color figure charges

- Immediate publication on acceptance

- Inclusion in PubMed, CAS, Scopus and Google Scholar

- Research which is freely available for redistribution

Submit your manuscript at 